\title{
¿Existe en la constipación idiopática severa un trastorno difuso de la motilidad intestinal?
}

\author{
Ana María Madrid S, Carlos Defilippi C. \\ Disturbances of small intestinal \\ motility in patients with chronic \\ constipation
}

Background: Studies in patients with chronic severe constipation, suggest the presence of a diffuse motor disorder of the gastrointestinal tract. Aim: To investigate small bowel motor activity in a group of patients with severe constipation. Material and Methods: Forty three patients (age range 13 to 70 years, 40 women) with severe constipation referred to our motility laboratory, where studied. Ten had a previous colectomy. Radiological examinations showed a megacolon in 30 and dilatation of small intestinal loops in nine; the remaining $\mathrm{X}$ rays studies were normal. Small intestinal motility was studied using perfused catheters and external transducers. Results: Ten patients had normal motor recordings. Eight patients showed a neuropathic disorder characterized by a continuous irregular pattern of contractions of normal amplitude, with absence of phase III of the migrating motor complex (MMC). Sixteen presented a normal MMC associated to a decreased amplitude of contractions (Miopatic disorder). Nine showed both types of motor disorders (Mixed pattern). Normal radiological findings were more commonly associated to normal manometric recordings. By contrast, an altered motor activity, mainly of myopathic and mixed type motor disorder, was observed in all patients with dilated small intestinal and colonic loops. Conclusions: The presence of small intestinal motor disorders was a frequent finding in this selected group of patients with chronic constipation (Rev Méd Chile 2006; 134: 181-6).

(Key words: Constipation; Gastrointestinal motility; Intestine, small)

Recibido el 27 de enero, 2005. Aceptado el 4 de agosto, 2005.

Sección de Gastroenterología, Departamento de Medicina, Hospital Clínico Universidad de Chile. Santiago de Chile.

L a constipación es una manifestación clínica frecuente, que se ha definido por la presencia de menos de 3 evacuaciones semanales, sin embargo, recientemente se han agregado como criterios: el esfuerzo defecatorio, características de las deposiciones y otros ${ }^{1-5}$.

Correspondencia a: Ana María Madrid S. Hospital Clínico U. de Chile - Sección Gastroenterología, Santiago, Chile. Teléfono: (56-2) 6788350. Fax: (56-2) 6788349.

E-mail: amadrid@ns.hospital.uchile.cl
En ausencia de alteraciones anatómicas obstructivas se han reconocido tres tipos de trastornos en la constipación crónica: a) Tránsito colónico normal, que corresponde en general a una sobrestimación del síntoma por parte de pacientes con alteraciones psicológicas. b) Constipación con tránsito lento, denominada también inercia colónica, que puede estar relacionada con alteraciones ya sea de la musculatura colónica 0 bien de la inervaciónn,7. c) Constipación por alteraciones del mecanismo de la defecación, 
mencionada en la literatura con los términos de disinergía pelviana o anismus, en la cual existe una disfunción del puborectalis o del esfínter anal externo ${ }^{8,9}$. Su diagnóstico se basa en algunos estudios de laboratorio, como la electromiografía y defecografía, exámenes que son de difícil interpretación ${ }^{1,9}$. No es infrecuente la superposición de estos trastornos fisiopatológicos.

Esta clasificación fisiopatológica está basada principalmente en la distribución de marcadores radiopacos administrados por vía oral. Los estudios directos de la motilidad colónica en estos pacientes son escasos, ya que sólo en los últimos años los avances tecnológicos han consentido la realización de registros confiables y prolongados en humanos y establecer su relación con los movimientos del contenido colónico ${ }^{10-12}$. Recientemente se ha confirmado en registros de $24 \mathrm{~h}$ una disminución de la actividad motora colónica en pacientes con inercia colónica ${ }^{13}$.

En enfermos con constipación por tránsito lento refractarios a la terapia médica, se ha utilizado la colectomía como recurso terapeútico. Sin embargo, los resultados de esta cirugía no siempre han sido satisfactorios ya que hasta $70 \%$ de los pacientes continúan teniendo dificultad para defecar, distensión y dolor abdominal, sin embargo, existen marcadas diferencias en las series publicadas ${ }^{14-16}$.

Estos resultados insatisfactorios junto con otras evidencias, sugieren que algunos enfermos pudieran presentar en forma asociada alteraciones de segmentos más proximales del tubo digestivo.

Existen investigaciones que apoyan la presencia de alteraciones de la motilidad esofágica, del vaciamiento gástrico y un retardo del tránsito del intestino delgado en estos pacientes ${ }^{17,18}$. Otros autores han podido objetivar alteraciones de la motilidad antroduodenal que sugieren la presencia de un trastomo difuso de motilidad del tubo digestivo ${ }^{19-21}$. El interés de estos estudios es poder determinar si las variaciones de la motilidad gastroduodenal pudieran tener un valor predictivo sobre los resultados de la colectomía, en pacientes con inercia colónica. Sin embargo, en general los estudios realizados han mostrado resultados controversiales.

Los objetivos de este trabajo fueron: 1) Presentar la experiencia de nuestro laboratorio en el estudio de la motilidad del intestino delgado, en un grupo seleccionado de pacientes con constipación idiopática severa de larga evolución. 2)
Establecer las características clínicas y radiológicas en este grupo de pacientes. 3) Correlacionar los hallazgos manométricos con alteraciones radiológicas a nivel del intestino delgado y grueso.

\section{PACIENTES Y MÉTODOS}

Ingresaron al estudio 43 pacientes (40 mujeres), con un promedio de edad de 39,4 años (rango 13 a 77 años) con el diagnóstico de constipación crónica severa, que fueron referidos a nuestro laboratorio de motilidad desde diferentes centros, entre los años 1995 y 2003. En 33 pacientes la constipación se presentaba desde la infancia y en los restantes tenía una duración promedio de 12,3 años (rango 1 a 30). El número de evacuaciones fue en promedio de 1 cada 11,7 días. Diez pacientes habían sido sometidos, previo al estudio, a tratamiento quirúrgico para la constipación consistente en colectomía total (9 casos) y hemicolectomía (1 caso), un enfermo fue sometido a dos intervenciones quirúrgicas. En todos los pacientes se obtuvo información de exámenes radiológicos efectuados en su unidad de origen, tanto de intestino delgado, como de colon (enema baritada), incluyendo aquellos efectuados antes de la colectomía. En 12 pacientes el estudio radiológico de intestino delgado y grueso fue informado como normal. Nueve tenían estudio radiológico de intestino delgado con dilatación de asas. En 30 enfermos una enema baritada fue informada como megacolon. La coexistencia de ambas alteraciones se observó en 8 casos. El estudio de tránsito intestinal con marcadores radiopacos se realizó sólo en 18 pacientes, 15 de ellos tenían en el control radiológico $80 \%$ o más de los marcadores al 5o día, distribuidos a lo largo del marco colónico (constipación por tránsito lento) y en los restantes fue normal.

Manometría intestinal. La técnica utilizada ha sido descrita previamente ${ }^{22}$. Brevemente: se utilizó un conjunto de 4 catéteres de polivinilo con un diámetro interior de $0,9 \mathrm{~mm}$, con puntos de registro separados $3 \mathrm{~cm}$ entre sí, perfundidos mediante un sistema neumohidráulico (Arndorfer Medical Specialty Greendele, Wiscosin, U.S.A) y conectados a un polígrafo (Nihon Kohden Co., Tokyo, Japón).

Procedimiento. Se suspendió el uso de fármacos procinéticos y antibióticos $72 \mathrm{~h}$ previas al estu- 
dio. Después de un ayuno de $12 \mathrm{~h}$, se pasaron los catéteres manométricos al estómago y bajo visión fluoroscópica, se avanzó el extremo distal hasta el ángulo de Treitz, quedando los puntos de registro entre la tercera porción del duodeno y el ángulo de Treitz. Luego se realizó un registro por un mínimo de $5 \mathrm{~h}$ con el paciente en decúbito dorsal.

Análisis de datos. La frecuencia y amplitud de las contracciones fueron medidas a través de un programa de computación especialmente diseñado. La identificación de las diferentes fases del complejo motor migratorio (CMM) se realizó directamente en el papel poligráfico de acuerdo a las siguientes características: en la fase I se observa ausencia de actividad motora, la fase II se identifica por la presencia de contracciones aparentemente irregulares y la fase III por contracciones regulares de $12 \mathrm{cpm}$ con una duración mayor a $3 \mathrm{~min}$ y de carácter propagado. Los valores normales de las contracciones: amplitud $32 \pm 5,6$ $\mathrm{mm}$ de $\mathrm{Hg}$ y frecuencia 1,2 $\pm 0,2 \mathrm{cpm}$ fueron definidos según un estudio previo en un grupo de voluntarios sanos ${ }^{22}$. Se calculó además un índice de motilidad intestinal IMI cuyo valor es equivalente al producto del promedio de la amplitud por la frecuencia de las contracciones.

Los trazados se separaron en cuatro grupos: a) normal, caracterizado por la presencia de una o más fases III del CMM, amplitud y frecuencia promedio de las contracciones con diferencias menores de 2 DE del grupo normal; b) miopáticos: promedio de amplitud de las contracciones de la fase II disminuido (diferencia mayor a 2 DE del promedio del grupo normal) c) neuropáticos: ausencia de fases III del CMM con contracciones de amplitud normal d) mixto: una combinación de ambas alteraciones, miopáticas y neuropáticas.

Estadística. Los resultados se expresan como promedio \pm desviación estándar. Para la comparación de los resultados de los estudios manométricos entre diferentes grupos se utilizó el análisis de varianza. Para comparar los hallazgos radiológicos y manométricos se empleó el test de Chi cuadrado. Las diferencias se consideraron estadísticamente significativas con un valor de $p$ $<0,05$.

\section{RESULTADOS}

En sólo 10 pacientes se registraron parámetros normales de motilidad intestinal. Ocho enfermos presentaron alteraciones manométricas que permitieron definirlos como grupo neuropático. En éstos se registró durante todo el período de estudio una actividad motora irregular, sin fases III del CMM y con contracciones de amplitud en rangos normales. Sin embargo, debido a una menor frecuencia de las ondas, se observó una disminución significativa del IMI (Tabla 1).

La mayor parte de los pacientes fueron catalogados como miopáticos: en todos estuvo presente una actividad cíclica del CMM con una o más fases III. En ellos se observó una disminución de 33,5\% de la amplitud de las contracciones en relación con el grupo normal. Si bien la frecuencia no fue diferente, la menor amplitud determinó una disminución significativa del IMI (Tabla 1). La amplitud de las contracciones de la fase III fue significativamente menor en comparación con el grupo normal.

Nueve pacientes presentaron alteraciones mixtas caracterizadas por total ausencia de la actividad cíclica, asociada a una reducción de la amplitud de las contracciones, a la que se asoció una caída significativa del IMI (Tabla 1).

En 6 de 12 pacientes con radiología de intestino delgado y grueso normales, la motilidad intestinal fue también normal. En cambio sólo en 4 de 22 pacientes con megacolon y en ninguno de los 8 pacientes con dilatación de ambos segmentos la actividad motora del intestino delgado fue normal. Hay diferencias significativas entre radiología normal y este último grupo de pacientes. Por otro lado, ningún paciente con radiología nomal presentó alteraciones motoras de tipo mixto, en cambio éstas se observaron en 6 pacientes con megacolon ( $p<0,05)$ y en 3 con dilatación difusa del tubo digestivo. La frecuencia de aparición de las alteraciones neuropáticas, miopáticas y mixtas se observa en la Figura 1.

De los 10 pacientes sometidos a colectomía, todos se encontraban sintomáticos al momento de efectuarse el estudio de motilidad intestinal: en 9 se observó persistencia de la constipación, en 6 distensión y dolor abdominal, y en 3 manifestaciones sugerentes de suboclusión intestinal. En estos enfermos se observaron los 4 tipos de trastorno motor distribuidos en forma relativamente similar. 
Tabla 1. Características de la motilidad del intestino delgado en ayunas en pacientes con constipación crónica

\begin{tabular}{|c|c|c|c|c|c|c|c|c|}
\hline & $\mathrm{n}$ & Edad & $\overline{\mathrm{x}}$ & $\begin{array}{c}\text { Fase III } \\
\overline{\mathrm{x}} / \mathrm{h}\end{array}$ & $\begin{array}{c}\text { Amplitud } \\
\text { (mmHg) }\end{array}$ & $\begin{array}{c}\text { Amplitud } \\
\text { (mmHg) }\end{array}$ & $\begin{array}{l}\text { Fase II } \\
\text { Frecuencia } \\
\text { cpm }\end{array}$ & IMI \\
\hline Normal & 10 & $40,9 \pm 18$ & $2,1 \pm 1$ & $0,4 \pm 0,2$ & $31,1 \pm 11$ & $26,5 \pm 6,1$ & $1,1 \pm 0,2$ & $28,5 \pm 6$ \\
\hline Neuropático & 8 & $38,8 \pm 14$ & - & - & - & $31,6 \pm 17$ & $0,6 \pm 0,4$ & $19,2 \pm 17 *$ \\
\hline Miopático & 16 & $36,8 \pm 16$ & $2,4 \pm 1$ & $0,5 \pm 0,3$ & $24,6 \pm 6^{*}$ & $17,8 \pm 2,6$ & $0,8 \pm 0,5$ & $14,1 \pm 9 *$ \\
\hline Mixto & 9 & $35,8 \pm 17$ & - & - & - & $17,1 \pm 3$ & $0,5 \pm 0,3$ & $8,5 \pm 6^{*}$ \\
\hline
\end{tabular}

IMI= índice de motilidad intestinal. $\bar{x}=$ promedio de fases III por estudio. $\bar{x} / \mathrm{h}$ promedio de fases III por hora. $* p<0,05$.

\section{DisCUSIÓN}

En una serie de pacientes con constipación crónica, demostramos en la mayoría de ellos alteraciones de la motilidad del intestino delgado, siendo el trastorno de carácter miopático el más frecuentemente observado. Ya que estos pacientes portadores de una constipación desde la infancia o de larga data y severa, fueron referidos a nuestro laboratorio, desde diferentes servicios y centros habiendo sido algunos de ellos sometidos previamente a cirugía, no se contó con toda la información clínica y de laboratorio deseable. De tal manera que sólo 18 tenían estudio de tránsito colónico y no tenemos información sobre manometría rectal, test de expulsión del balón 0 defecografía, con los cuales pudiera haberse descartado un trastorno de la evacuación. Sin embargo, en la mayoría de aquellos en que se realizó evaluación del tránsito, el estudio fue sugerente de inercia colónica.

Resulta sorprendente que sólo 10 enfermos fueran catalogados como normales. Esto pudiera estar relacionado con el hecho de que se trata de un grupo de pacientes muy seleccionado y con un cuadro clínico severo.

El grupo de pacientes neuropáticos se caracterizó por presentar ausencia de actividad cíclica durante las cinco horas de registro, si bien este período ha sido estimado adecuado para este análisis por diferentes autores, ha existido en la literatura una controversia al comparar los resultados así obtenidos, con registros de 24 $\mathrm{h}^{23}$. Recientemente un estudio automatizado de motilidad del intestino delgado de $24 \mathrm{~h}$, en pacientes con constipación crónica, demostró alteraciones nocturnas de la actividad cíclica del $\mathrm{CMM}$, en aproximadamente un tercio de los pacientes 24

La alteración motora más frecuentemente observada en estos pacientes fue la presencia de algún grado de compromiso miopático presente en $58,1 \%$ de los estudios, ya sea en forma aislada o acompañando a un trastorno neuropático. Estos resultados contrastan con otros estudios ${ }^{19,21,24}$ en los cuales no se describen alteraciones miopáticas 0 éstas se observan en forma ocasional. No es fácil explicar estas diferencias, sin duda, la selección de los pacientes y los criterios en el análisis manométrico, pueden haber influido, sin embargo, todos estos pacientes presentaron además un IMI disminuido. El IMI no fue utilizado como criterio para la incorporación a los diferentes grupos. Se observó además, en los miopáticos una disminución de la amplitud de las contracciones en fase III.

A pesar que los estudios radiológicos en estos pacientes fueron efectuados en diferentes centros, son interesantes sus hallazgos y su relación con la motilidad: cuando tanto la radiología del intestino delgado como la del colon fueron normales, el resultado más frecuente fue una manometría normal. Cuando sólo se observó megacolon, situación predominante, los trastornos motores se distribuyeron en igual proporción entre miopáticos, neuropáticos y mixtos. En 


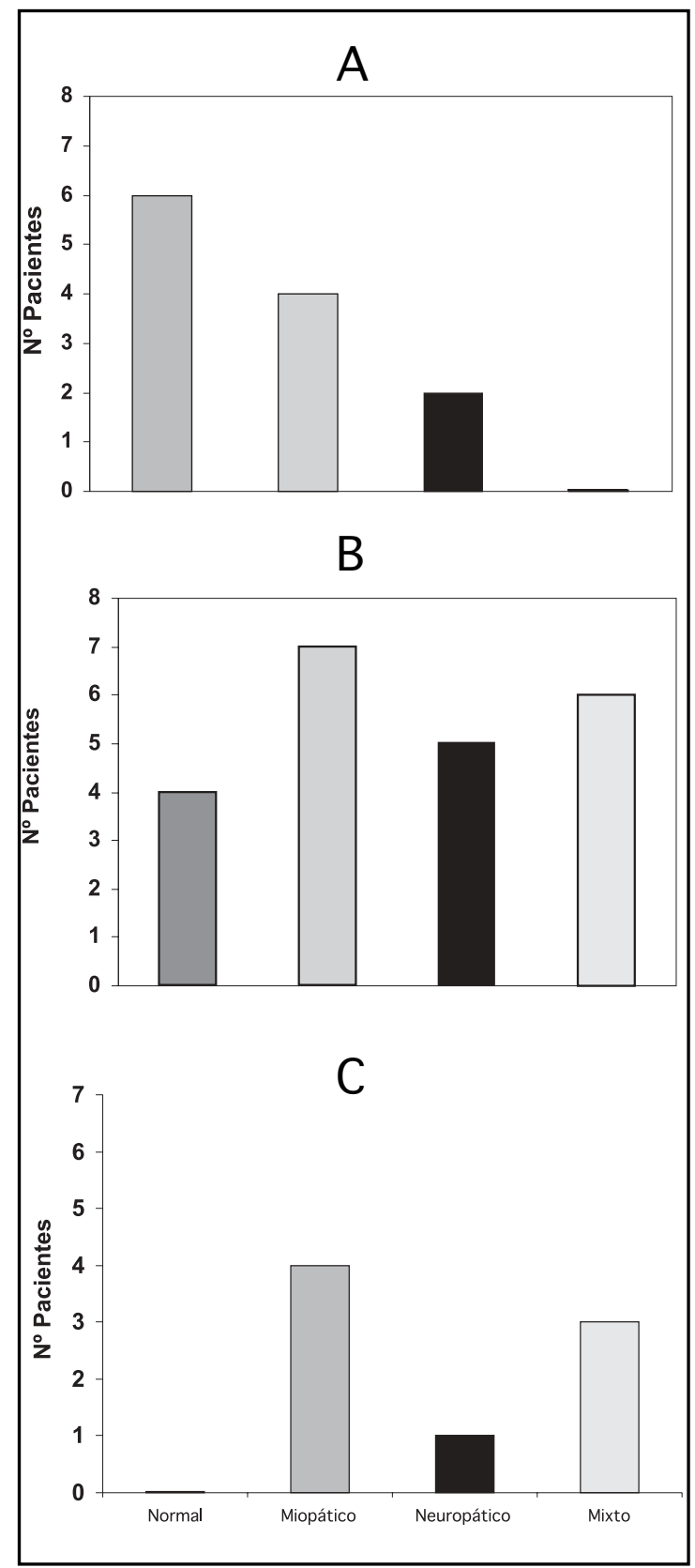

FIGURA 1. Relación entre hallazgos radiológicos y manométricos. Número de pacientes con manometría intestinal normal o con las diferentes alteraciones miopáticas, neuropáticas y mixtas, en presencia de: radiología de intestino delgado y colon normales $1 \mathrm{~A}$, megacolon 1B y dilatación de intestino delgado y megacolon 1C.

${ }^{*} \mathrm{p}<0,05: \mathrm{A}$ v/s C, **A v/s B. cambio cuando ambos, intestino delgado y colon, estaban dilatados, casi todos los pacientes presentaron un trastorno motor de tipo miopático. Este último grupo puede corresponder a pacientes que están cursando con un síndrome de pseudobstrucción intestinal. Sin embargo, no todos los pacientes con pseudobstrucción intestinal presentan dilatación de asas intestinales, 0 bien ésta ocurre en forma intermitente ${ }^{25}$. En estos enfermos la posibilidad de estar en presencia de un cuadro de carácter progresivo, hace recomendable un seguimiento adecuado, evitando en ellos, en los períodos de crisis, una laparotomía exploradora por la sospecha de obstrucción intestinal.

Cabe considerar que, si bien la dilatación de asas intestinales está primariamente relacionada con el trastorno motor y una alteración de la propulsión del contenido, la dilatación de éstas modifica a su vez la motilidad por razones técnicas, al ejercer una menor acción sobre los catéteres manométricos ${ }^{26}$.

Estimamos que la motilidad del intestino delgado en estos pacientes con constipación, pudiera constituirse en un factor predictor de los resultados del tratamiento quirúrgico, con el fin de efectuar una mejor selección. Glia y $\operatorname{col}^{27}$ efectuaron un seguimiento de 5 años a un grupo de 17 enfermos con colectomía total por constipación de tránsito lento. Estos autores observaron mejores resultados en presencia de una manometría de intestino delgado normal, comparada con aquellos en que la manometría estuvo alterada.

A la luz de nuestros hallazgos manométricos sería necesario realizar un trabajo prospectivo analizando, como en el trabajo anterior, con un número mayor de observaciones, el resultado de la colectomía en un grupo seleccionado de estos enfermos. Sin embargo, frente a un grado de mayor compromiso de la actividad motora del intestino delgado, en especial en aquellos pacientes con trastornos mixtos o con grados más severos de compromiso miopático, sería prudente analizar en forma muy cuidadosa la alternativa quirúrgica. 


\section{REFERENCIAS}

1. Thompson WG, Longstreth G, Drossman D, Heaton $K$, IRVINE EJ, MÜLER-LISSNER S. Functional bowel disorders and functional abdominal pain. En Drossman DA ed. Rome II The Functional Gastrointestinal Disorders. Laurence KS, Allen Press 2000; 351-432.

2. RAO S. Constipation: evaluation and treatment. Gastroenterol Clin N Am 2003; 32: 659-83.

3. Lembo A, Camueri M. Chronic Constipation. N Engl J Med 2003; 349: 1360-8.

4. Higgins P, Johanson J. Epidemiology of constipation in North America: A systematic Review. Am J Gastroenterol 2004; 99: 750-?

5. KАмM MA. Constipation and its management. Options go beyond laxatives and include behavioural treatment as well as new drugs. BMJ 2003; 327: 459-60.

6. Bharucha AE, Phiшips SF. Slow transit constipation. Gastroenterol Clin N Am 2001; 30: 77-95.

7. EL-SalHY M. Chronic idiopathic slow transit constipation: pathophysiology and management. Colorectal Disease 2003; 5: 288-96.

8. Schrank B, Herold A, Leiss O. Functional outlet obstruction: Anismus, spastic pelvic floor syndro$\mathrm{me}$, and dyscoordination of the voluntary sphincter muscles. Scand J Gastroenterol 1999; 5: 449-53.

9. D'Hoore A, PenNinckX F. Obstructed defecation. Colorectal Dis 2003; 5: 280-7.

10. Bampton PA, Dinning PG, Kennedy ML, LubowsKi DZ, Cоок IJ. Prolonged multi-point recording of colonic manometry in the unprepared human colon: Providing insight into potentially relevant pressure wave parameters. Am J Gastroenterol 2001; 96: 1838-48.

11. Rao S, Sadeghi P, Beaty J, Kavlock R, Ackerson K. Ambulatory 24-h colonic manometry in healthy humans. Am J Physiol 2001; 280: G629-G639.

12. Cook IJ, Furukana Y, Panagopoulos V, Cowns P, DENT J. Relationships between spatial patterns of colonic pressure and individual movements of content. Am J Physiol 2000; 278: G329-G341.

13. Hagger R, Kumar D, Benson M, Grundy A. Colonic motor activity in slow-transit idiopathic constipation as identified by 24-h pancolonic ambulatory manometry. Neurogastroenterol Motil 2003; 15: 515-22.

14. Pikarsky AJ, Singh JJ, Weiss E, Nogueras J, Wexner SD. Long-term follow-up of patients undergoing colectomy for colonic inertia. Dis Colon Rectum 2001; 44: 179-83.
15. Ghosh S, Papachry sostomou M, Batool M, EastwooD MA. Long-term results of subtotal colectomy and evidence of noncolonic involvement in patients with idiopathic slow-transit constipation. Scand J Gastroenterol 1996; 31: 1083-91.

16. Nylund G, Oresiand T, Fasth S, Nordgren S. Longterm outcome after colectomy in severe idiopathic constipation. Colorectal Dis 2001; 3: 253-8.

17. Altomare DF, Portincasa P, Rinaldi M, Di Ciaula A, Martinew E, Amoruso A et al. Slow-transit constipation. A solitary symptom of a systemic gastrointestinal disease. Dis Colon Rectum 1999; 42: 231-40.

18. Van Der Sijp JR, Kamm MA, Nightingale JM, Britton KE, GranowsKa M, Mather SJ et al. Disturbed gastric and small bowel transit in severe idiopathic constipation. Dig Dis Sci 1993; 38: 837-44.

19. Kumar D, Waldron D, Wimams NS, Wingate DL. Slow-transit constipation: a pan enteric motor disorder? Gastroenterology 1989; 96: A277.

20. Bassotti G, Stanghemini V, Chiarioni G, Germani U, De Giorgio R, Vantin I et aL. Upper gastrointestinal motor activity in patients with slow-transit constipation. Further evidence for an enteric neuropathy. Dig Dis Sci 1996; 41: 1999-2005.

21. GLA A, LINDBeR G. Antroduodenal manometry findings in patients with slow-transit constipation. Scand J Gastroenterol 1998; 33: 55-62.

22. Madrid AM, Hurtado C, Venegas M, Cumsiue F, DEFILPPI C. Long-term treatment with cisapride and antibiotics in liver cirrhosis: Effect on small intestinal motility, bacterial overgrowth and liver function. Am J Gastroenterol 2001; 96: 1251-5.

23. Camileri M, Hasier WL, Parkman HP, Quigley EMM, SoFFER E. Measurement of gastrointestinal motility in the Gl laboratory. Gastroenteroloy 1998; 115: 747-62.

24. Scott SM, Picon L, Knowles CH, Fourquet F, Yazaki E, Wiшams NS et al. Automated quantitative analysis of nocturnal jejunal motor activity identifies abnormalities in individuals and subgroups of patients with slow transit constipation. Am J Gastroenterol 2003; 98: 1123-34.

25. Di LoRENZo C. Pseudo-obstruction: Current Approaches. Gastroenterology 1999; 116: 980-7.

26. HaNSEN MB. Small intestinal manometry. Physiol Res 2002; 51: 541-56.

27. Glia A, AKerlund JE, LuNdBerg G. Outcome of colectomy for slow-transit constipation in relation to presence of small-bowel dysmotility. Dis Colon Rectum 2004; 47: 96-102. 